\title{
Extrato da Glândula de Feromônio na Atração e Estimulação Alimentar de Ninfas de Podisus nigrispinus (Dallas) e Supputius cincticeps (Stal)
}

\author{
Jorge B. Torres ${ }^{1}$, José C. Zanuncio ${ }^{2,4}$, Jorge L.D. Saavedra ${ }^{2}$ e Jeffrey R. Aldrich ${ }^{3}$ \\ ${ }^{1}$ Departamento de Agronomia e Fitossanidade, Av. Dom Manoel de Medeiros \\ s/n, Dois Irmãos, UFRPE, 52171-900, Recife, PE. \\ ${ }^{2}$ Departamento de Biologia Animal, UFV, 36571-000, Viçosa, MG. \\ ${ }^{3}$ USDA-ARS, Insect Chemical Ecology Laboratory, Beltsville, MD, USA 20705. \\ ${ }^{4}$ Autor correspondente.
}

An. Soc. Entomol. Brasil 26(3): 463-469 (1997)

Pheromone Gland Extracts of Podisus nigrispinus (Dallas) and Supputius cincticeps (Stal) as Attractant and Feeding Stimulant for Nymphs

ABSTRACT - The attractant and feeding stimulant effects of extracts of dorsal abdominal glands of Podisus nigrispinus (Dallas) and Supputius cincticeps (Stal) (Heteroptera: Pentatomidae) added to nymphs' diet were evaluated and compared with stimulant and feeding effects on factitious prey. The extract spread over the diet attracted $88 \%$ of nymphs of $P$. nigrispinus. Similar results were achieved for nymphs feeding on diet with extract (spread over and added - 84\%), and using the factitious prey Musca domestica L. (Diptera: Muscidae) $(100 \%)$. Nymphs of $S$. cincticeps feeding on the same diet, showed maximum value of 60 and $56 \%$ but lower than that achieved on the factitious prey, Tenebrio molitor L. (Coleoptera: Tenebrionidae) (92\%). Nymphs of P. nigrispinus and $S$. cincticeps showed similar lower weights when reared on the artificial diet ( 2.7 and $1.2 \mathrm{mg}$ ), respectively, or when reared with extract added to the diet (2.8 and $1.6 \mathrm{mg}$ ), extract spread over the diet $(2.1$ and $1.0 \mathrm{mg}$ ), or with extract spread over and added to the diet $(2.4$ and $1.3 \mathrm{mg})$ than that on the factitious prey (3.1 and $3.0 \mathrm{mg}$ ).

KEY WORDS: Insecta, predatory bugs, mass rearing.

RESUMO - Estudou-se o efeito do extrato de glândulas dorso-abdominais, produtoras de feromônio sexual de Podisus nigrispinus (Dallas) e de Supputius cincticeps (Stal) (Heteroptera: Pentatomidae), adicionadas em dieta artificial, como atrativo e estimulante alimentar para ninfas desses predadores, comparado com ninfas criadas somente em dieta artificial ou com presas alternativas. $\mathrm{O}$ extrato de glândulas pincelado sobre a dieta artificial, apresentou atratividade em $88 \%$ de ninfas de $P$. nigrispinus alimentando-se desta dieta, semelhante àquelas sobre dieta pincelada e contendo o extrato em mistura (10\% v/p) (84\%) e aquelas alimentadas com a presa alternativa Musca domestica L. (Diptera: Muscidae) (100\%). S. cincticeps, apresentou 60 e 56\% de ninfas alimentandose sobre as mesmas dietas, o que foi menor que a percentagem de ninfas alimentando-se na presa alternativa Tenebrio molitor L. (Coleoptera: 
Tenebrionidae) (92\%). Ninfas de P. nigrispinus e de S. cincticeps, apresentaram pesos semelhantes, com a dieta artificial sem mistura (2,7 e 1,3 mg), com extrato de glândula $(2,8$ e $1,6 \mathrm{mg})$, pincelada com o extrato $(2,1 \mathrm{e} 1,0 \mathrm{mg})$ e com a dieta artificial com extrato de glândula e pincelada com o mesmo (2,4 e 1,2 mg), respectivamente. No entanto, esses pesos, foram inferiores àqueles obtidos com a presa alternativa $(3,1$ e $3,0 \mathrm{mg})$.

PALAVRAS-CHAVE: Insecta, percevejos predadores, criação massal.

Feromônios sexuais de pentatomídeos tem sido estudados, com ênfase para Nezara viridula (L.) (Aldrich et al. 1987, Baker et al. 1987, Borges et al. 1987, Borges 1995). Atualmente, têm-se buscado a utilização destes compostos para predadores desta família, como Podisus maculiventris (Say) e Perillus bioculatus (F.), nos EUA (Aldrich 1995) e Podisus nigrispinus (Dallas) e Supputius cincticeps (Stal), no Brasil (Aldrich et al. 1997). Esses feromônios são considerados a principal razão da ampla dispersão desses insetos nos agroecossistemas, devido à grande quantidade dessas substâncias produzidas por glândulas dorsoabdominais dos machos (Aldrich 1995), que atraem adultos e ninfas.

P. nigrispinus e $S$. cincticeps estão sendo criados em dieta artificial com melhores resultados para $P$. nigrispinus, mantido por gerações contínuas em dieta artificial (Saavedra et al. 1995) sem redução na fecundidade (Saavedra et al. 1992a, Zanuncio et al. 1996). No geral, P. nigrispinus, $S$. cincticeps, $P$. maculiventris e Podisus sagitta (Fab.) (=P. nigrispinus) criados com dieta artificial tiveram sua fase ninfal prolongada de $10-15 \%$ e o peso de adultos reduzido de 15 - 20\%, em comparação com aqueles criados com a presa viva (De Clercq \& Degheele 1992, Saavedra et al. 1992a, Zanuncio et al. 1996), indicando que essas dietas apresentam condições para o desenvolvimento e reprodução desses predadores, mas que podem ser melhoradas. A aceitação das dietas pelas ninfas tem apresentado problemas com conseqüente redução da alimentação nas mesmas, por não apresentarem movimentos, que são estímulos utilizados pelos predadores para a localização da presa (Pfannenstiel et al. 1995) e, também, porque as "lagartas" artificiais não exalam substâncias utilizadas como cairomônios.

Devido à eficiência de atração do feromônio, liberado pelas glândulas dorsoabdominais de $P$. nigrispinus e $S$. cincticeps, para ninfas e adultos dessas espécies, objetivou-se verificar a melhoria da aceitação da dieta artificial por ninfas de $P$. nigrispinus e $S$. cincticeps, adicionando-se extratos destas glândulas às dietas.

\section{Material e Métodos}

Dois bioensaios foram instalados, utilizando glândulas de feromônio sexual, extraídas de machos de $P$. nigrispinus e de $S$. cincticeps. Os insetos foram provenientes da colônia mantida em laboratório, onde são alimentados com larvas de Musca domestica L. (Diptera: Muscidae) e Tenebrio molitor L. (Coleoptera: Tenebrionidae), respectivamente. Foram extraídas glândulas de 10 machos de cada espécie e maceradas em 5,0 $\mathrm{ml}$ de água destilada. Não foi utilizado solvente, comumente empregado nesse tipo de extração, uma vez que o extrato de glândulas foi incorporado à dieta e poderia alterá-la.

A dieta artificial foi a de Saavedra et al. (1992b) para P. nigrispinus e de Zanuncio et al. (1996/97) para $S$. cincticeps. As ninfas foram separadas no início do segundo estádio e mantidas em placas de Petri $(15 \times 1,5 \mathrm{~cm})$, com um chumaço de algodão umedecido no seu interior. Após 24 h nesse estádio, sem alimentação, foi retirado o algodão umedecido 
e oferecido às ninfas, em grupos de cinco por placa de Petri, dieta artificial envolta com Parafilm-M. As placas de Petri foram mantidas a $26 \pm 2{ }^{\circ} \mathrm{C}, 60 \pm 5 \%$ UR e $14 \mathrm{~h}$ de fotofase. Os tratamentos consistiram de: $\mathrm{T}_{1}$ "lagartas" de dieta artificial; $\mathrm{T}_{2}$ - "lagartas" de dieta artificial com $10 \%$ (v/p) do extrato de glândulas; $\mathrm{T}_{3}$ - "lagartas" de dieta artificial pinceladas com extrato de glândulas; $\mathrm{T}_{4}$ "lagartas" de dieta artificial com 10\% (v/p) do extrato de glândulas e pincelada; $\mathrm{T}_{5}$ testemunha, empregou-se pupas de $M$. domestica para P. nigrispinus e de T. molitor para $S$. cincticeps. Foram utilizadas cinco repetições com cinco ninfas cada, com avaliação a cada cinco minutos e até $120 \mathrm{~min}$, registrando-se o início da alimentação das mesmas. Além disso, ninfas vivas, após $24 \mathrm{~h}$ de exposição foram pesadas para avaliação do consumo de dietas. O número de ninfas, que iniciou a alimentação na dieta, foi submetido à análise de regressão para o tempo de chegada, enquanto a percentagem máxima de ninfas que iniciaram a alimentação e o peso de ninfas após $24 \mathrm{~h}$, foi submetido à análise de variância e aplicado o teste de Scott-Knott a $\mathrm{P}<0,05$.

\section{Resultados e Discussão}

Todas as ninfas de $P$. nigrispinus e $92 \%$ das ninfas de $S$. cincticeps alimentaram-se das presas alternativas $M$. domestica e T. molitor, após 90 e $50 \mathrm{~min}$, respectivamente (Figs. 1, 2). A maior percentagem de ninfas, alimentando-se na dieta artificial, foi obtida nos tratamentos com extrato da glândula pincelado sobre a dieta ou quando este foi misturado a dieta artificial e pincelado sobre a mesma, tanto para P. nigrispinus (88 e 84\%) como para $S$. cincticeps (60 e 56\%) (Tabela 1). O extrato das glândulas atraiu as ninfas que foram induzidas a iniciar a alimentação na dieta artificial, melhorando a aceitação da mesma por esses predadores. Isto é importante, pois tem sido relatado menor atratividade de predadores para dietas artificiais do que para presas alternativas (Cohen 1985, Saavedra et al. 1995).
Para as presas alternativas, foi observada continuidade de alimentação, o que não ocorreu nos tratamentos com dieta e extratos de glândulas. Ao empregar-se o extrato pincelado sobre a "lagarta" artificial, tanto para $P$. nigrispinus quanto para $S$. cincticeps, houve incremento significativo na percentagem de localização e início de alimentação pelas ninfas (Figs. 1, 2). Porém, isto não foi mantido, obtendo-se, após 120 min de avaliação, percentagem semelhante de ninfas em alimentação, tanto no tratamento com dieta artificial como naqueles com pinceladas do extrato de glândulas sobre as "lagartas" artificiais. Desta forma, pode-se considerar que a adição do extrato de glândula na dieta sugere que não tenha ocorrido efeito estimulante alimentar, como observado na continuidade de alimentação nas presas alternativas mas melhorou o início de alimentação das ninfas dos predadores na dieta artificial. No entanto, para P. nigrispinus, quando utilizou-se extrato de glândula (10\% $\mathrm{v} / \mathrm{p}$ ), as ninfas alimentaram-se por período de tempo maior e percentagem maior, quando comparado a $S$. cincticeps (Figs. 1 e 2). Isto pode estar relacionado ao melhor desempenho de $P$. nigrispinus nesta dieta (Saavedra et al. 1995) e não devido a adição do extrato da glândula. A aceitação do alimento pelo predador é realizada rapidamente, através das papilas nervosas do aparelho bucal (Cohen 1990), o que indica a influência do extrato da glândula para o inicio da alimentação. Estes resultados, para a permanência da alimentação, provavelmente, representam a tendência, também, nos estádios mais avançados de $P$. nigrispinus e $S$. cincticeps, pois as enzimas digestivas, nas fases de ninfa e adulta, são semelhantes, indicando semelhança nas necessidades nutricionais e no comportamento de percevejos predadores nessas fases (Tauber \& Tauber 1987, Stamopoulos et al. 1993).

Para todos os tratamentos com dieta artificial houve mortalidade de ninfas (Tabela 1), embora consideravelmente menor para $P$. nigrispinus comparado a $S$. cincticeps. Mortalidade de ninfas de 2 o estádio foi, 
Tabela 1. Percentagem (média \pm EP) de ninfas em alimentação, peso (média \pm EP) e sobrevivência de ninfas de 2 o estádio de Podisus nigrispinus e de Supputius cincticeps, após $24 \mathrm{~h}$.

\begin{tabular}{|c|c|c|c|c|}
\hline \multirow[b]{2}{*}{ Tratamentos } & \multicolumn{2}{|c|}{ Podisus nigrispinus } & \multicolumn{2}{|c|}{ Supputius cincticeps } \\
\hline & $\begin{array}{l}\% \text { de Ninfas } \\
\text { Alimentando-se }\end{array}$ & $\begin{array}{l}\text { Peso em mg }{ }^{1} \\
\text { (\% de Sobrevivência) }\end{array}$ & $\begin{array}{c}\% \text { de Ninfas } \\
\text { Alimentando-se }\end{array}$ & $\begin{array}{c}\text { Peso em mg }{ }^{1} \\
(\% \text { de Sobrevivência) }\end{array}$ \\
\hline Dieta artificial (DA) & $52,0 \pm 18,3 \mathrm{c}$ & $2,7 \pm 0,25 b \quad(84,1)$ & $36,0 \pm 15,6 \mathrm{c}$ & $1,3 \pm 0,86 \mathrm{~b}$ \\
\hline $\begin{array}{l}\text { Extrato da glândula (EG) } \\
\text { misturado a dieta artificial }\end{array}$ & $72,0 \pm 10,2 b$ & $2,8 \pm 0,24 b \quad(88,0)$ & $32,0 \pm 13,6 \mathrm{c}$ & $1,6 \pm 0,81 b$ \\
\hline DA pincelada com EG & $88,0 \pm 14,2 \mathrm{a}$ & $2,1 \pm 0,43 b \quad(92,0)$ & $60,0 \pm 25,3 b$ & $1,0 \pm 0,31 b$ \\
\hline EG misturado a DA & $84,0 \pm 13,5 \mathrm{a}$ & $2,4 \pm 0,28 b \quad(92,0)$ & $56,0 \pm 15,3 b$ & $1,2 \pm 1,20 \mathrm{~b}$ \\
\hline Presa alternativa (pupa) ${ }^{2}$ & $100,0 \pm 0,0 \mathrm{a}$ & $3,1 \pm 0,17 \mathrm{a} \quad(96,0)$ & $92,0 \pm 5,2 \mathrm{a}$ & $3,0 \pm 1,20 \mathrm{a}(100)$ \\
\hline C.V. $(\%)$ & 14,3 & 13,5 & 23,1 & 15,2 \\
\hline
\end{tabular}

${ }^{1}$ Médias seguidas pela mesma letra, na coluna, não diferem entre si pelo teste de Scott-Knott a $5 \%$ de probabilidade.

${ }^{2}$ Pupas de Musca domestica para Podisus nigrispinus e de Tenebrio molitor para Supputius cincticeps.

também, observada por Saavedra et al. (1992b), com P. nigrispinus e por Zanuncio et al. (1996/97) com $S$. cincticeps, em dieta artificial. Este fato é também destacado para outros predadores como Geocoris punctipes (Say) e Chrysopa spp. (Cohen \& Urias 1988,

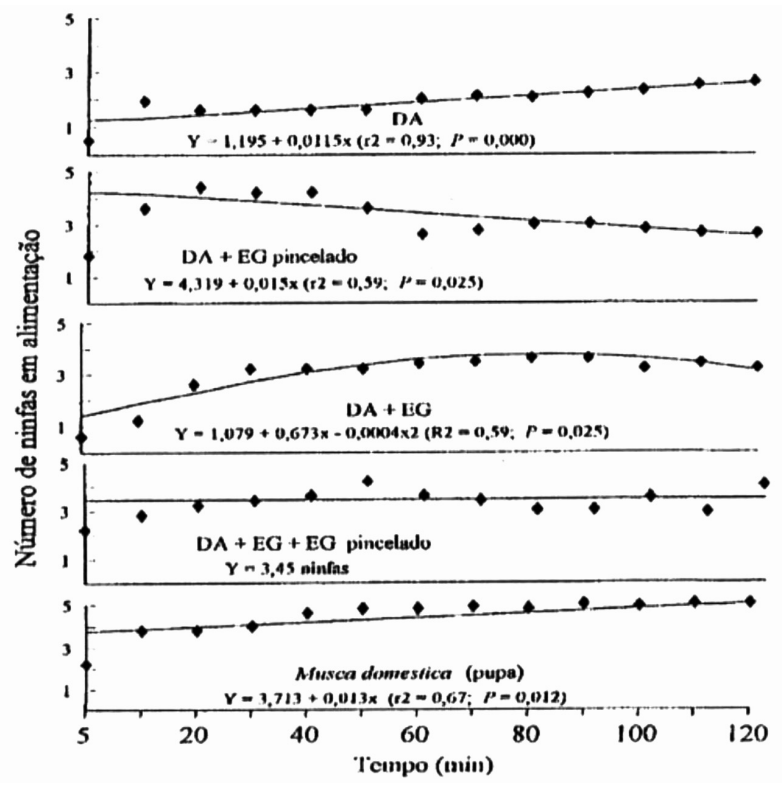

Figura 1. Número médio de ninfas de 2otádio de Podisus nigrispinus que iniciaram a alimentação em dieta artificial (DA); em DA pincelada com extrato da glândula (EG) de ferômonio (DA + DG); em DA + EG de feromônio misturado à dieta (DA + DG); em DA + EG + EG pincelado sobre a "lagarta" artificial e, em pupas de Musca domestica. 
Cohen 1990, Milbrath et al. 1993) que apresentam dificuldades de adaptação a dieta artificial no $2^{\circ}$ estádio. dieta artificial, tem apresentado capacidade reprodutiva semelhante à obtida em presas alternativas (De Clercq \& Degheele 1992,

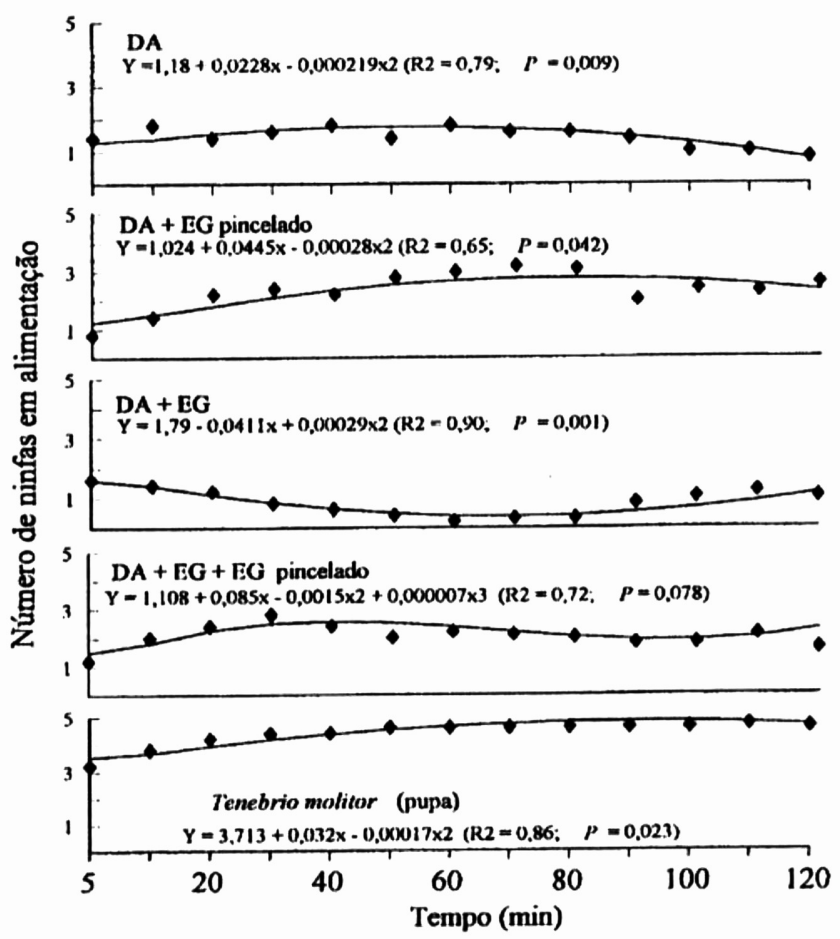

Figura 2. Número médio de ninfas de 2o estádio de Supputius cincticeps que iniciaram a alimentação na dieta artificial (DA); em DA pincelada com extrato da glândula (EG) de ferômonio (DA + DG); em DA + EG de feromônio misturado à dieta (DA + DG); em DA + EG + EG pincelado sobre a "lagarta" artificial e, em pupas de Tenebrio molitor.

O peso de ninfas caracteriza que estas não se alimentaram, em igual intensidade nas dietas artificiais e nas presas alternativas, apresentando peso semelhante em todas as dietas, porém inferior àquele obtido na presa alternativa, para ambas espécies (Tabela 1). Menor peso de predadores alimentados com dietas artificiais é comum (Cohen 1985, De Clercq \& Degheele 1992, Saavedra et al. 1992a, Saavedra et al. 1995), sendo, supostamente, conseqüência da menor quantidade de alimento ingerido e menor metabolismo (Cohen \& Urias 1988). No entanto, adultos de menor peso, obtidos de
Saavedra et al. 1992a, Saavedra et al. 1995, Zanuncio et al. 1996). Este estudo mostra novas possibilidades para a melhoria da aceitação de dietas artificiais por percevejos predadores. Como as ninfas iniciaram, mas não continuaram a alimentação na dieta artificial (Figs. 1, 2), isto sugere que novas pesquisas devam investigar a adequação da concentração do extrato de glândula a ser adicionado à dieta artificial.

A utilização de extrato da glândula de feromônio, pode ser recomendada para a melhoria da aceitação da dieta artificial por ninfas de $P$. nigrispinus e, em menor escala, 
para ninfas de $S$. cincticeps.

\section{Agradecimentos}

Ao Dr. Miguel Borges, do CENARGEN/ EMBRAPA e aos Professores Marcelo C. Picanço e Raul C. N. Guedes, da Universidade Federal de Viçosa, pelas sugestões na elaboração desse manuscrito. Ao BIOAGRO, CNPq e FAPEMIG, pelas bolsas e auxílios concedidos.

\section{Literatura Citada}

Aldrich, J.R. 1995. Chemical communication in the true bugs and parasitoid exploitation, p. 318-363. In R.T. Carde \& W.J. Bell (eds.), Chemical Ecology of Insects II. New York, Chapman \& Hall, $433 p$.

Aldrich, J.R., J.E. Oliver, W.R. Lusby, J.P. Kochansky \& J.A. Lockwood. 1987. Pheromone strains of the cosmopolitan pest, Nezara viridula (Heteroptera: Pentatomidae). J. Exp. Zool. 214: 171175.

Aldrich, J.R., J.C. Zanuncio, E.F. Vilela, J.B. Torres \& R.D. Cave. 1997. Field tests of predaceous pentatomid pheromones and semiochemistry of Podisus species (Hemiptera: Pentatomidae: Asopinae). An. Soc. Entomol. Brasil 26:1-14.

Baker, R., M. Borges, N.G. Cooke \& R.N. Herbert. 1987. Identification and synthesis of (Z)-(1'S, 3'R, 4'S) (-)-2(3',4'-epoxy-4'-methylcyclohexyl)-6methylhepta-2,5-iene, the sex pheromone of the southern green stink bug, Nezara viridula (L.). Chem. Commun. 414-416.

Borges, M., P.C. Jepson \& P.E. Howse. 1987. Long-range location and closerange courtship behaviour of the stink bug, Nezara viridula and its mediation by sex pheromones. Entomol. Exp. Appl. 44:205-212.

Borges, M. 1995. Atractant compounds of the southern green stink bug, Nezara viridula (L.) (Heteroptera: Pentatomidae). An. Soc. Entomol. Brasil 24: 215-225.

Cohen, A.C. 1985. Simple method for rearing the insect predator Geocoris punctipes (Heteroptera: Lygaeidae) on a meat diet. J. Econ. Entomol. 78: 1173-1175.

Cohen, A.C. 1990. Feeding adaptations of some predaceous Hemiptera. Ann. Entomol. Soc. Am. 83: 1215-1223.

Cohen, A.C. \& N.M. Urias. 1988. Food utilization and egestion rates of the predator Geocoris punctipes (Hemiptera: Heteroptera) fed artificial diets with rutin. J. Entomol. Sci. 23: 174-179.

De Clercq, P. \& D. Degheele. 1992. A meatbased diet for rearing the predatory stinkbugs Podisus maculiventris and Podisus sagitta (Het.: Pentatomidae). Entomophaga 37: 149-157.

Milbrath, L.R., M.J. Tauber \& C.A. Tauber. 1993. Prey specifity in Chrysopa: an interespecific comparison of larval feeding and defensive behavior. Ecology 74: 1384-1393.

Pfannenstiel, R.S., R.E. Hunt \& K.V. Yeargan. 1995. Orientation of a hemipteran predator to vibrations produced by feeding caterpillars. J. Insect Behav. 8: 1-10.

Saavedra, J.L.D., J.C. Zanuncio, T.M.C. Della Lucia \& F.P. Reis. 1992a. Efeito da dieta artificial na fecundidade e fertilidade do predador Podisus connexivus Bergroth, 1891 (Hemiptera: Pentatomidae). An. Soc. Entomol. Brasil 21:69-76. 
Saavedra, J.L.D., J.C. Zanuncio, T.M.C. Della Lucia \& E.F. Vilela. 1992b. Dieta artificial para crianza de Podisus connexivus (Hemiptera: Pentatomidae). Turrialba 42: 258-261.

Saavedra, J.L.D., J.C. Zanuncio, E.F. Vilela, C.S. Sediyama \& P. De Clercq. 1995. Development of Podisus nigrispinus (Dallas) (Heteroptera: Pentatomidae) on meat-based artificial diets. Med. Fac. Landbouww. Univ. Gent 60: 683-688.

\section{Stamopoulos, D.C., G. Diamantidis \& A.} Chloridis. 1993. Enzymatic activities registered in gut tissues of Podisus maculiventris (Hem.: Pentatomidae). Entomophaga 38: 493-499.

Tauber, C.A. \& M.J. Tauber. 1987. Food specificity in predaceous insects: a comparative ecophysiological and genetics study. Evol. Ecol. 1: 175-186.

Zanuncio, J.C., J.L.D. Saavedra, T.V. Zanuncio \& G.P. Santos. 1996/97. Dessarrolo y reprodución de Supputius cincticeps (Heteroptera: Pentatomidae) en dieta artificial por dos generaciones. Rev. Biol. Trop. 44/45:247-251.

Recebido em 25/03/96. Aceito em 17/09/97. 